\title{
Effects of reinforcement-paired stimuli on general activity
}

\author{
THOMAS S. HYDE \\ Mather Memorial Building, Case Western Reserve University, Cleveland, Ohio 44106 \\ and \\ MILTON A. TRAPOLD \\ University of Minnesota, Minneapolis, Minnesota 55455
}

\begin{abstract}
Two experiments were performed to explore the mechanisms responsible for the increase in activity that occurs in response to stimuli which have been paired with reinforcement ( $S^{R}$ ). The first experiment showed a sharp increase in activity to $\mathbf{S}^{\mathbf{R}}$-paired stimuli under conditions in which subjects were not required to perform any instrumental response to obtain the $\mathbf{S}^{\mathbf{R}}$. This result seemed to rule out reinforcement of instrumental "food getting" behavior as the mechanism responsible for the learned activity increases. A second experiment used an "omission training" procedure to further explore the mechanisms underlying the activity increase. In this experiment, $\mathbf{S} \mathbf{R}$ was omitted on those trials on which activity increases were present during the stimulus. In this condition, no increases in activity were observed during the stimulus. There was, however, the characteristic increase in activity in a yoked-control group which received the same number and distribution of stimulus-paired $S^{R_{S}}$. The results of the second experiment open the possibility that increases in activity to $S^{R}$-paired stimuli could be due to the adventitious reinforcement of motor behavior rather than the Pavlovian conditioning of a motivational state.
\end{abstract}

A number of investigators have established that stimuli which have been paired with positive reinforcement come to produce an increase in general activity (Bindra \& Palfai, 1967; Sheffield \& Campbell, 1954; Zamble, 1967). The most frequent interpretation of these results has been that some motivational state becomes classically conditioned to the reinforcement-paired stimuli and that conditioned motivational state, or "excitement," then energizes motor activity (Bindra \& Palfai, 1967; Sheffield, 1966; Zamble, 1967). Sheffield (1966) has relied on such an interpretation in his "Drive Induction" theory of instrumental learning.

Others have specifically argued against such a conditioned motivation explanation of the activity-increasing effects of reinforcement-paired stimuli. Baumeister, Hawkins, and Cromwell (1964) and Bolles (1963), for example, have pointed to the possibility that these effects could be due to the adventitious reinforcement of motor activity during the stimulus change which precedes reinforcement. Any activity occurring during the stimulus would be reinforced and its probability thereby increased during future stimulus presentations.

Either, or both, of these above explanations could be applied to the results of two recent experiments which showed activity increases to reinforcement-paired

The authors wish to thank Kent Eaton for his assistance in running the subjects in the first experiment and J. Bruce Overmier for his most helpful comments on an earlier draft of this report. Requests for reprints should be sent to Thomas $S$. Hyde, Department of Psychology, Mather Memorial Building, Case Western Reserve University, Cleveland, Ohio 44106. stimuli. Zamble (1967) simply paired a 10-sec auditory stimulus with the delivery of a food pellet. He found that with repeated stimulus-reinforcement pairings, there was an increase in activity during the stimulus which he attributed to the classical conditioning of a motivational state to the stimulus. It seems equally possible, however, that the increase in activity simply reflected the learning of an instrumental approach response to the food magazine during the stimulus.

Bindra \& Palfai (1967) made a specific attempt to rule out such an instrumental learning interpretation in their experiment. They performed the stimulus-reinforcement pairings in an apparatus which severely restricted the movement of their subjects. When later tested in a larger enclosure, subjects showed the expected increase in activity during the reinforcement-paired stimulus. They argued that because very little movement was possible during the stimulus-reinforcement pairings, the increases in activity could be best interpreted as the classical conditioning of "incentive motivation" to the stimulus. The possibility remains, however, that some movement, however slight, could have been reinforced during the pretraining sessions and then generalized to the test situation. Even though the movement of their subjects was restricted, they still had to make an active instrumental response to obtain reinforcement from the magazine. Even if this "magazine response" were small in magnitude, one cannot preclude the possibility that its generalization to the test situation produced the observed increases in activity.

The following two experiments were designed to study the nature of activity changes to 


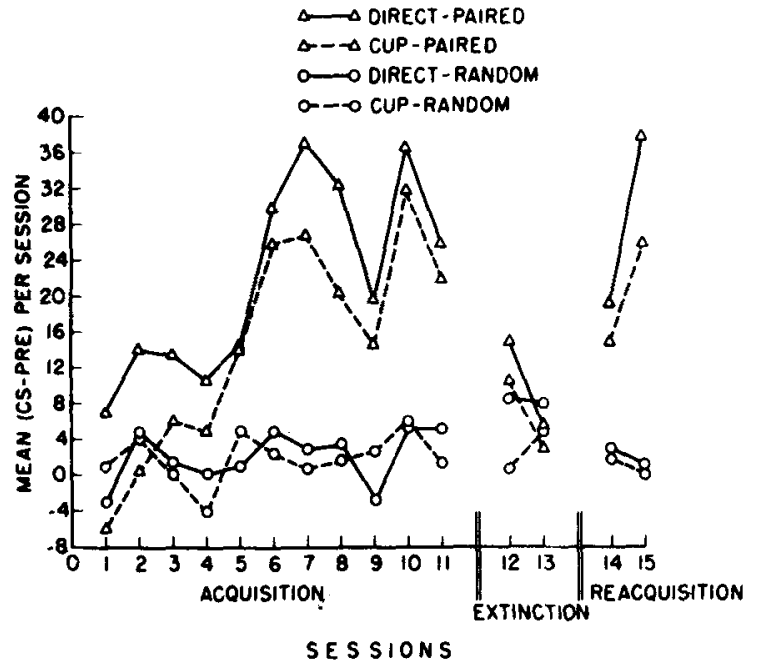

Figure 1. The mean increase in activity level during the stimulus for each treatment group on each session of Experiment I.

reinforcement-paired stimuli, and, in particular, to study whether these changes are due to the classical conditioning of motivational states or the learning of instrumental responses to the reinforcement-paired stimuli.

\section{EXPERIMENT I}

The first experiment was designed to study the effects of reinforcement-paired stimuli on activity in a situation where no instrumental response was necessary to obtain reinforcement. The experimental design specifically compares the effects of reinforcement-paired stimuli using two different methods of reinforcement delivery-one which requires the usual magazine response and another where subjects do not have to make any instrumental response to obtain reinforcement. This latter condition is made possible by a surgical preparation developed by Gross, Trapold, and Hyde (1968) which allows sucrose reinforcement to be delivered directly to the mouth of an unrestrained rat.

\section{Method}

Subjects and apparatus. Subjects were 16 male Simonson albino rats, approximately 120 days old at the beginning of the experiment. All subjects were surgically prepared following the Gross et al. (1968) procedure. Briefly, this technique consists of attaching the hub of a long (3-in.) hy podermic needle to the top of the skull by the screw-and-dental-acrylic method commonly used to attach brain electrodes. The needle portion is bent so that it runs outside the skin down the midline of the face and into the mouth between the incisors.

All training was conduced in a cylindrical Plexiglas chamber $15 \mathrm{in}$. in diam and $18 \mathrm{in}$. high. The chamber was mounted on an activity measuring platform described by Davis and Ellison (1964). On one side of the chamber, a metal cup was attached to the wall, approximately 2 in. from the floor. The chamber and activity measuring platform were enclosed in a sound-attenuated box.
Liquid reinforcement consisted of $.075 \mathrm{ml}$ of $12 \%$ sucrose solution delivered by a Davis Model LR-120 liquid pump. A piece of polyethylene tubing ran from the pump, over a pulley system, and down to the rat inside the chamber. Permanently affixed to the end of the polyethylene tubing was a waterproof swivel-joint modeled after that described by Epstein and Teitelbaum (1962) but modified so that its output end was a female Luer-Lok fitting, which fit directly over the hypodermic needle mounted on the rat's skull. Half of the subjects received liquid reinforcement delivered directly into their mouths via the polyethylene tube. The other half of the subjects were also hooked up to the tubing but actually received their reinforcement via the metal cup on the wall of the chamber.

Procedure. All subjects were allowed 2 weeks to recover from the surgical preparation. Following recovery, subjects were reduced to $80 \%$ of their ad-lib body weight and placed on a $23-\mathrm{h}$ feeding schedule.

Following three 40-min adaptation sessions, subjects were randomly divided into four treatment conditions. Group 1 (direct-paired) had a 10-sec tone (approximately 1,000 Hz) paired with sucrose reinforcement delivered directly into the mouth. Group 2 (cup paired) had the 10-sec tone paired with reinforcement delivered into the metal cup located on the chamber wall. Group 3 (direct random) received sucrose reinforcement delivered into their mouths and tone presentations, but these two events were programmed to occur independently and randomly in time. Group 4 (cup random) received tone presentations and cup-delivered reinforcements in a random fashion.

All subjects were given 11 consecutive days of training. During each 40-min training session, all subjects received 20 tone presentations with a mean interstimulus interval of $2 \mathrm{~min}$. Subjects also received 20 reinforcements per session. For the two paired groups ( 1 and 2 ), the sucrose reinforcement was programmed to coincide with the offset of the 10-sec tone. For the two random groups ( 3 and 4 ), the 20 reinforcements were programmed to occur with no temporal relationship to the auditory stimulus, thus conforming to Rescorla's (1967) "truly random control" procedure.

Following this initial 11 days of acquisition training, all subjects were given an additional 2 days of extinction. During each 40-min extinction session, the tone was presented 20 times with a mean interstimulus interval of $2 \mathrm{~min}$. No sucrose reinforcements were delivered during extinction.

Following extinction, subjects were given 2 days of reacquisition training, where the initial treatment conditions were reinstated for all subjects. Throughout the experiment, the level of activity was measured during a 10-sec period immediately preceding each tone presentation and during the tone presentation itself.

\section{Results and Discussion}

Data for the experiment consisted of the difference in activity measures between the $10 \mathrm{sec}$ period immediately preceding each stimulus presentation and the 10-sec stimulus period. Figure 1 presents the mean of these difference scores for each group on each day of testing.

Over the initial 11 days of training, the two paired groups (1 and 2), which had the auditory stimulus paired with reinforcement, showed greater increases in activity during the tone than did the two random control groups (3 and 4). There were no appreciable differences between the two paired groups nor between the two random control groups. A two-way repeated measures ANOVA (treatment groups by test sessions) on the 
initial 11 days of testing yielded a significant effect for treatment conditions $(\mathrm{F}=18.68, \mathrm{df}=3 / 12, \mathrm{p}<.01)$ and for sessions $(F=3.21, \mathrm{df}=10 / 112, \mathrm{p}<.01)$. The Treatment Group by Test Session interaction did not reach statistical significance. Scheffé contrasts on individual treatment means yielded the following: Group 1 (direct paired) differed from Group 3 (direct random), $\mathrm{F}=12.60, \mathrm{df}=3 / 12, \mathrm{p}<.01$; Group 1 (direct paired) differed from Group 4 (cup random), $F=13.38$; $\mathrm{df}=3 / 12, \mathrm{p}<.01$; Group 2 (cup paired) differed from Group 3 (direct random), $F=4.44, d f=3 / 12, p<.05$; and Group 2 (cup paired) differed from Group 4 (cup random), $F=4.89, \mathrm{df}=3 / 12, \mathrm{p}<.05$. None of the other Scheffe comparisons reached statistical significance.

During the two extinction sessions, the differences between paired and random treatment conditions were greatly reduced and then reappeared during the two final reacquisition sessions. A two-way repeated measures ANOVA (treatments by sessions) on the two extinction sessions failed to show any statistically significant effects. A two-way repeated measures ANOVA on the two final reacquisition sessions yielded a statistically significant effect for treatment conditions $(F=4.77$, $\mathrm{df}=3 / 12, \mathrm{p}<.05$ ). In this final analysis, the sessions effect and the Sessions by Treatment interaction did not reach statistical significance.

The results suggest that reinforcement-paired stimuli can produce an increase in activity whether or not subjects are required to make an instrumental response to obtain reinforcement. The group which had reinforcement delivered directly into their mouths, in fact, showed the largest increase in activity during the stimulus. This finding would seem to minimize the possibility that activity increases during reinforcement-paired stimuli are due to the reinforcement of instrumental "food getting" behavior as might have been possible in the Bindra and Palfai (1967) and Zamble (1967) experiments. The results also indicate that increases in activity to reinforcement-paired stimuli show reliable extinction and reacquisition effects.

\section{EXPERIMENT II}

Although it was arranged to minimize the amount of required "food getting" behavior, the first experiment does not rule out the possibility that subjects may have learned some other kind of instrumental behavior during the reinforcement-paired stimulus. It seems highly possible that any behavior patterns which occur during a reinforcement-paired stimulus would increase in probability during future stimulus presentations. Since rats are reasonably active after being habituated to an experimental apparatus, it is likely that a high level of gross motor activity would occur during a $10-\mathrm{sec}$ stimulus and be adventitiously reinforced. Neither direct reinforcement, as used in the first experiment, nor physical restraint, as used in the Bindra and Palfai (1967) experiment, would completely rule out this possibility.

In the second experiment, an attempt was made to preclude the possibility of a reinforcement contingency for motor activity by failing to reinforce subjects if they showed anticipatory increases in activity level during the stimulus. This is the "omission training" procedure used by Sheffield (1965) to isolate classically conditioned factors in salivary conditioning.

\section{Method}

Subjects and apparatus. Subjects were 12 male Simonson albino rats, approximately 120 days old at the beginning of the experiment. The apparatus, surgical preparation, and deprivation schedule were the same as those used in the first experiment. All subjects received sucrose reinforcement delivered directly into their mouths.

Procedure. After a food deprivation schedule was established and subjects were adapted to the experimental chamber, they were randomly divided into three treatment conditions. Subjects in Group 1 (reverse contingency) received 20 tone presentations during each 40-min session. If subjects showed a higher level of activity during the tone than during the immediately preceding 10-sec period, reinforcement failed to occur. If the activity was the same or lower than during the immediately preceding interval, the stimulus was followed by sucrose reinforcement delivered directly into the mouth. The stimuli, reinforcement, and interstimulus intervals were the same as those in the first experiment. In Group 2 (yoked), each of the four subjects failed to receive reinforcement on the same trials as did the subject to whom they were yoked in the first group. In this group, failure to receive reinforcement was, of course, independent of their activity levels during the stimulus. In Group 3 (random control), subjects received 20 tone presentations and 20 sucrose reinforcements per session in such a way that there was no temporal contingency between the two events.

All subjects were given 28 days of training and an additional 4 days of extinction where no sucrose reinforcements were delivered. The level of activity was measured both during the 10 -sec period immediately preceding each stimulus presentation and during the stimulus presentation itself.

\section{Results and Discussion}

As in the first experiment, the data represent the differences between activity measured during the stimulus and during the immediately preceding 10 -sec period. Results are presented in Figure 2, which gives the mean difference scores for each group on each day of the experiment. As is apparent in Figure 2, only Group 2 (yoked) showed an increase in activity during the reinforcement-paired stimulus. A two-way repeated measures ANOVA (treatment by sessions) on the initial 28 days of acquisition revealed a statistically significant effect for treatment conditions $(F=48.36$, $\mathrm{df}=2 / 9$, $\mathrm{p}<.01)$, for sessions $(\mathrm{F}=1.96, \mathrm{df}=27 / 243, \mathrm{p}<.01)$, and for the Treatment by Sessions interaction $(F=1.47$, $\mathrm{df}=54 / 243, \mathrm{p}<.05)$. Scheffé contrasts on individual group means showed that Group 2 (yoked) showed greater increases in activity during the stimulus than did Group 1 (reverse) and Group 3 (random). The contrasts were $F=13.93(\mathrm{df}=2 / 9, \mathrm{p}<.01)$ and $F=21.45$ 


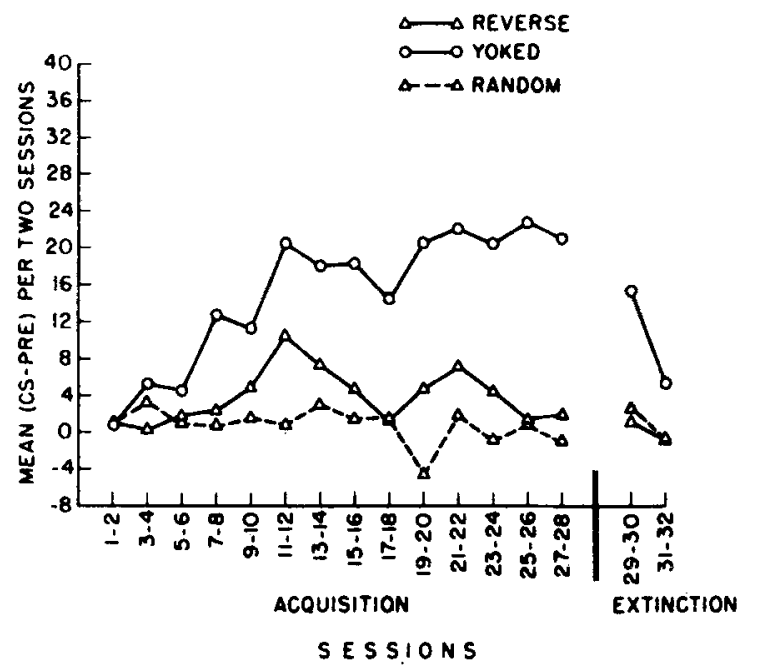

Figure 2. The mean increase in activity level during the stimulus for treatment group on each session of Experiment II.

$(\mathrm{df}=2 / 9, \mathrm{p}<.01)$, respectively. None of the other possible group differences reached statistical significance.

A second two-way repeated measures ANOVA (groups by sessions) was performed on the final 2 days of extinction. There were no statistically significant effects.

The results of the second experiment indicate that increases in activity during reinforcement-paired stimuli could quite possibly be due to the reirforcement of superstitious motor behavior. In the treatment condition where reinforcement was omitted on those trials where activity increases occurred during the stimulus, there was no reliable increase in activity over the 28 daily training sessions. Further, subjects in this condition did not differ from subjects in the random control group, where there was no contingency between the stimulus and reinforcement. Subjects in the yoked control group, on the other hand, who received the same number and distribution of reinforcements as subjects in the reverse contingency group did show the characteristic increase in activity during the stimulus.

\section{GENERAL DISCUSSION}

The results of the first experiment suggest that a direct reinforcement procedure which requires no "magazine response" can lead to an increase in activity during a reinforcement-paired stimulus. It is still possible, even with a procedure which does not require an instrumental response to obtain reinforcement, that activity increases are due to the adventitious reinforcement of instrumental behavior rather than to the Pavlovian conditioning of incentive motivation. Given the typically high level of motor activity shown by rats, some pattern of active responding would seem more likely to be reinforced during the reinforcement-paired stimulus than would "sitting still."

In the second experiment, where the contingencies were arranged to specifically omit reinforcement when there was an increase in activity during the stimulus, there was no reliable increase in activity during the reinforcement-paired stimulus. While this result seems to cast some doubt on the "conditioned motivation" interpretation suggested by Bindra and Palfai (1967) and Zamble (1967), there are at least two possible interpretations of this experimental result which would be consistent with the motivational view. First, it might be argued that the omission procedure used in the second experiment resulted in such a low frequency of CS-US pairings that the conditioned motivation extinguished, or at least remained at a very low level, insufficient to produce increases in activity. This contention does not seem supported, however, given the high levels of activity found in the yoked-control group.

Second, it might be argued that the omission training procedure introduced a reinforcement contingency for behavior which was physically incompatible with motivation-produced activity changes. It could be argued, for example, that the omission procedure simply led to the learning of behavior which did not effect the activity measuring apparatus. While this does not seem to occur when other classically conditioned behaviors are subjected to omission procedures (e.g., Sheffield, 1965), it could certainly be argued that conditioned activity is more likely to be "interfered with" by learned instrumental behavior than are other classically conditioned responses (e.g., salivation).

In summary, the first experiment obtained increased activity levels to reinforcement-paired stimuli under conditions where subjects were not required to perform an instrumental response to obtain reinforcement, and therefore, could be taken to support the view that such activity changes are due to the Pavlovian conditioning of incentive motivation. From the results of a second experiment, which showed that an omission training procedure eliminated activity increases to reinforcement-paired stimuli, it could be argued that such activity increases are due to adventitiously reinforced motor behavior. Given alternative interpretations of this second experiment, however, a resolution of this question must await further experimentation.

\section{REFERENCES}

Baumeister, A., Hawkins, W. F., \& Cromwell, R. L. Need states and activity level. Psy chological Bulletin, 1964, 61, 438-453. Bindra, A., \& Palfai, T. Nature of positive and negative incentive-motivational effects on general activity. Journal of Comparative and Physiological Psychology, 1967, 63, 288-297.

Bolles, $R$. C. Effect of food deprivation on the rat's behavior in its home cage. Journal of Comparative and Physiological Psy chology, 1963, 56, 456-460.

Davis, J. A., \& Ellison, G. P. A general purpose activity recorder with variable sensitivity. Journal of the Experimental Analy sis
of Behavior, 1964, 7, 117-1 18. 
Epstein, A. N., \& Teitelbaum, P. A watertight swivel joint permitting chronic injection into animals. Journal of Applied Physiology, 1962, 17, 171-172.

Gross, D. M. Trapold, M. A., \& Hyde, T. S. A simple technique for delivering liquids directly to the mouth of an unrestrained rat. Journal of the Experimental Analysis of Behavior, 1968 , 11, 191-195.

Rescorla $R$. Pavlovian conditioning and its proper contro procedures. Psychological Review, 1967, 74, 71-80.

sheffield, F. D. Relation between classical conditioning and instrumental learning. In W. F. Prokasy (Ed.), Classical conditioning. New York: Appleton-Century-Crofts, 1965.
Sheffield, F. D. New evidence for the drive-induction theory of reinforcement. In $\mathrm{R}$. $\mathrm{N}$ Haber (Ed), Current research in motivation. New York: Holt, Rinehart, and Winston, 1966.

Sheffield, F. D., \& Campbell, B. A. The role of experience in the "spontaneous" activity of hungry rats. Journal of Comparative and Physiological Psychology, 1954, 47, 97-100.

Zamble, E. Classical conditioning of excitement anticipatory to food reward. Journal of Comparative and Physiological Psy chology, 1967, 63, 526-529.

(Received for publication July 3, 1974; revision received March 14,1975 .) 